\title{
Modeling and Simulation of Wake Safety Interval for Paired Approach Based on CFD
}

\author{
Xin He, ${ }^{1}$ Yilong $\mathrm{Ma}^{2}$ Hong Yang $\mathbb{D},{ }^{3}$ and Yaqing Chen $\left.{ }^{3}\right)^{3}$ \\ ${ }^{1}$ Air Traffic Management College, Civil Aviation Flight University of China, Guanghan 618307, China \\ ${ }^{2}$ Hope College, Southwest Jiaotong University, Chengdu 610400, China \\ ${ }^{3}$ Civil Aviation Flight Technology and Flight Safety Research Base, Civil Aviation Flight University of China, \\ Guanghan 618307, China \\ Correspondence should be addressed to Yaqing Chen; chenyaqingmail@sina.com
}

Received 16 July 2021; Accepted 29 November 2021; Published 30 December 2021

Academic Editor: Chi-Hua Chen

Copyright (c) 2021 Xin He et al. This is an open access article distributed under the Creative Commons Attribution License, which permits unrestricted use, distribution, and reproduction in any medium, provided the original work is properly cited.

\begin{abstract}
In order to relieve the stress caused by the surge of flight flow, Closely Spaced Parallel Runways (CSPRs) have been built in many hub airports, and a paired approach mode has been applied to CSPRs in some countries. This paper proposes a method for optimizing the wake separation between aircrafts which utilizes a paired approach, aiming at reducing longitudinal separation by using computational fluid dynamics technology. Firstly, the model of the wake vortex field of the paired lead aircraft is constructed. Secondly, the numerical simulation preparation for the characteristics of the wake vortex field is completed through the computational pretreatment of the model. Thirdly, a calculation model of wake safety interval based on paired approach operation is established. Finally, the proposed method shows its superiority comparing with other methods. This method realized visual analysis of wake vortex through optimization modeling based on computational fluid dynamics, contributing to increasing the capacity of the runway and improving the operation efficiency of an aerodrome.
\end{abstract}

\section{Introduction}

In recent years, the airline industry develops rapidly and plays an important role in the world economy. The increasing trend in civil aviation transportation has remained high for years, and airport capacity has begun to show signs of decline. Most aviation hub cities have plans to rebuild and expand airports, so some large hub airports began to build closely spaced parallel runways (CSPRs).

Regarding the operation of CSPRs, due to safety considerations, the wake interval standard for single-runway operation is still adopted in China for the time being, which has not stimulated the expansion advantage of CSPRs. However, the paired approach (PA) mode was proposed by American scholars. The PA concept is the one that leverages the real-time navigation and communication capabilities of the ADS-B equipage initiative to increase airport capacity by performing simultaneous dependent approaches to CSPRs $[1,2]$. This operation mode greatly increases the capacity of
CSPRs when successive aircrafts are approaching under instrument meteorological conditions, which greatly reduces the wake interval and avoids the wake influence caused by the lead aircraft, providing the operation of CSPRs with a new direction [3].

In 2000, The concept of "safety zone" was introduced by Landry and Pritchett through the research of the paired approach [4]. The safety zone, also called the protection zone, of the paired approach mode is shown in Figure 1. It is an area in which the paired aircrafts can maneuver operate, and separation assurance and wake avoidance are provided. The front boundary of safety zone called collision safety limit (CSL). The rear boundary of the safety zone called wake safety limit (WSL). The American Mitte Company proposed a $3^{\circ}$ offset paired approach, which can better avoid the risk of wake encounters using computational fluid dynamics [5]. This research result has played a great role in the research of the subsequent paired approach mode, which has provided theoretical guidance for a simultaneous instrument 


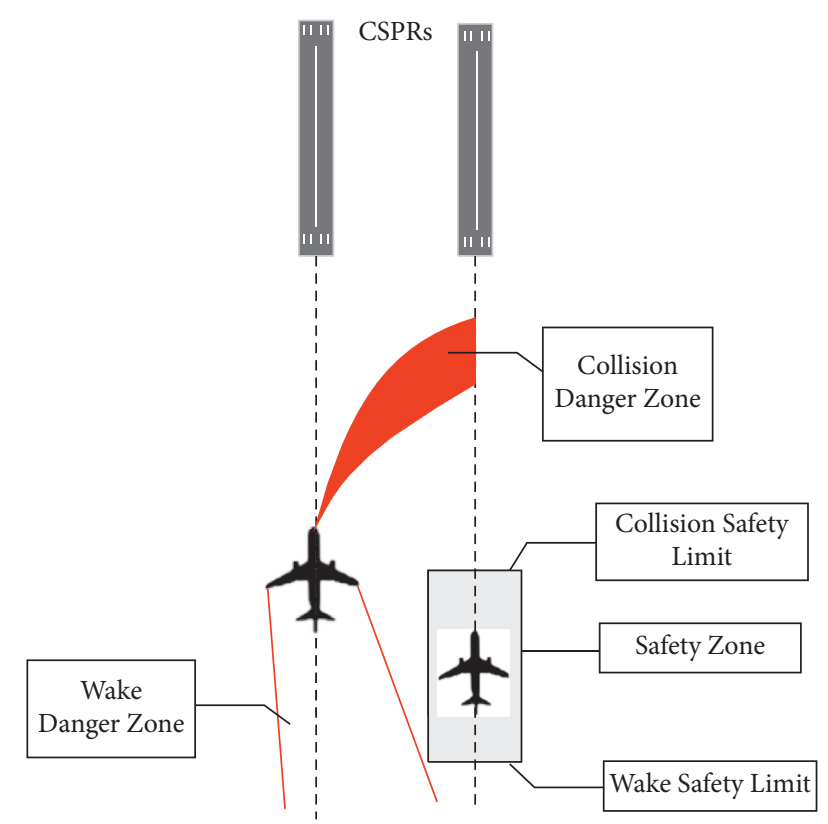

FIGURE 1: Schematic diagram of paired approach mode.

approach mode implemented at the airports such as Boston and San Francisco [6-8]. In 2001, the Advanced Aviation System Development Center of the company and the Industrial and System Engineering Campus of Georgia Tech University tested the initial procedures for the paired approach on a flight simulator, laying a foundation for the further definition of cockpit missions and the development of a cockpit separation management system [9].

NASA proposed to use the Monte Carlo method for the simulation calculation of the wake encounter risk caused by lead aircraft and proved the applicability of the method. The safety zone of the paired approach and the risk of collision during a wrong approach of the lead aircraft can also be simulated by using the Monte Carlo method [10-12]. In 2014, the Langley Research Center used the method of constructing a wake kinematics model to calculate the wake encounter risk for aircrafts that implemented the paired approach mode, which provided a reference for the quantitative analysis.

With the development of new technologies in civil aviation communications, navigation, surveillance equipment, and air traffic control, the operation of the paired approach mode has become more safe and efficient with the support of advanced equipment such as ADS-B surveillance equipment and the next-generation warning system ALAS. This has opened the door for the paired approach procedure based on interval management [13-16]. The Flight Research and Analysis Group of FAA began a safety study on the collision risk in 2017, associated with the lead aircraft's front boundary of the safety zone [2]. From 2018 to 2019, funded by NASA, MOSAIC ATM, United Airlines, and Honeywell, the research group jointly planned and conducted a paired approach mode flight test at San Francisco International Airport. The data obtained from the test will provide for the further implementation of the paired approach mode $[17,18]$.
This paper considered the safety of the paired approach mode, proposing a new method to study the wake safety interval of the paired aircrafts. We build the optimization model of the wake vortex field of the paired lead aircraft so that we can achieve visualized analysis of the wake influence. Compared with previous studies, the innovations of this paper are as follows:

Compared with experimental methods-wind tunnel test and water tunnel test, the CFD numerical simulation method shortens the research time, because it can rely on the rapid computing ability of the computer to build a vortex field model and carry out the numerical simulation, rather than constructing, testing, and maintaining the experimental equipment.

The CFD numerical simulation method not only improves the accuracy to a certain extent but also adapts to the numerical simulation of complex vortex fields.

Using the CFD method to study the safety zone of the paired approach wake is a breakthrough attempt, which provides new ideas and new solutions for using the fluid mechanics method to study the actual operation of civil aviation.

The authors' main contributions are summarized as follows: X.H. and Y.C. conceptualized and supervised the study; X.H. developed methodology and performed formal analysis; H.Y. collected data and wrote the original draft; Y.M. investigated and developed the model by software; Y.C. and X.H. validated the study; Y.C. collected resources and funds; all authors have read and agreed to the published version of the article.

\section{Research Method of Wake}

To determine the wake influence range of the paired approach aircraft, the motion characteristics of the wake vortex field of the paired aircraft must be analyzed and studied. At present, the research on the motion characteristics of aircraft wake vortex field mainly includes the following methods: wake feature detection test method, wake feature capture modeling method, and wake numerical simulation method. This section will discuss in detail the advantages and disadvantages of these methods.

2.1. The Test Method of Wake Feature Detection. The wake vortex has unique electromagnetic scattering characteristics, so it is easy to be effectively detected by sonar, radar, lidar, and other sensors [19]. Carrying out the wake feature detection test is of great value to the research and application of military and civil aviation.

Wind tunnel test is a conventional method to study the characteristics of the wake field of an aircraft. This method can measure the velocity distribution of the wake field in a certain space behind the aircraft model under different configurations. However, this method is subject to the influence of the test section scale and is mainly aimed at the detection of the characteristics of the wake field near the aircraft. The process of the wake generation and rolling up is shown in Figure 2 [20]. Measurement methods usually 


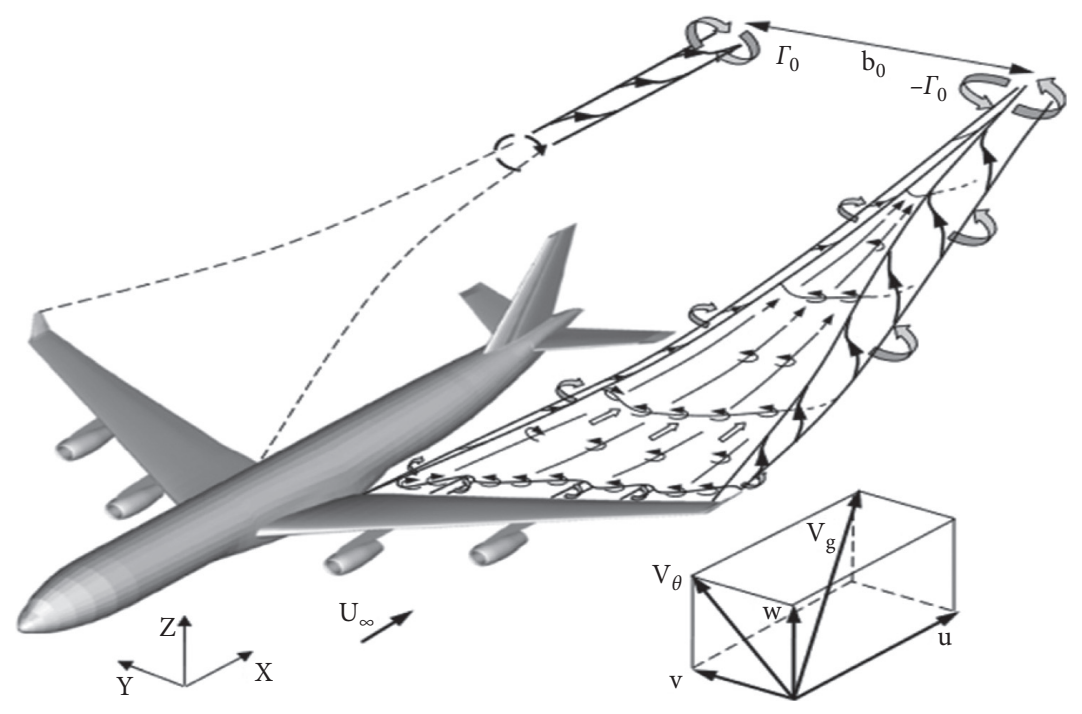

FIGURE 2: Wake generation and winding process.

include five-hole probe sensor measurement method [21], particle image velocimetry measurement method [22], or hot wire wind velocity method for measuring the wake field velocity over time. Since the 1970s, a large number of documents have recorded the velocity and vorticity fields of various configurations of typical transport aircraft measured by the wind tunnel test [23-31]. This method has the characteristics of good vortex field quality and high measurement accuracy, but it also has the following shortcomings: the wind tunnel structure is relatively complicated, the manufacturing cost is high, the experiment area is large, and it relies on high-precision sensors.

The water tunnel is a common test which uses water as the medium for dynamic experiments. The water tunnel test is more suitable for the experimental observation and research analysis of certain aerodynamic problems than the wind tunnel test, such as the generation and shedding of aircraft wingtip vortices [32]. The advantage of the water tunnel test is that the multiscale vortex structure near the wall, the wake vortex field shedding structure, and the spatial vortex separation structure can be clearly understood. The observed vortex field structure will not be interfered with by the tracer particle material properties, and the effect will be impaired $[33,34]$. In addition, the water tunnel experiment also has the following shortcomings: the water tunnel structure is relatively complex, the manufacturing cost is high, the research period is long, and it is easy to cause experimental system errors due to the interference of the subsystems in the vortex field and the influence of the fluid medium or model. Especially most experiments use scaled models for experiments, and the simulated vortex environment cannot be absolutely consistent with the actual working conditions.

2.2. The Method of Wake Feature Capture Modeling. In order to further simplify the analysis process and enhance the prediction effect, scholars have improved the modeling method of the wake vortex field based on the observations obtained from flight tests. Since these wake velocity field modeling methods are based on the theoretical analysis methods of fluid mechanics, they are very effective for some uncomplicated vortex problems. However, for some more complex and nonlinear governing equations, they are beyond reach. Thus, it is suitable for qualitative analysis of simple vortex problems or complex fluid mechanics problems.

2.2.1. Lift Line Theory and Wake Vortex Hypothesis. From the classic Prandtl lift line theory, it can be known that if the wingspan of an airplane wing is constant, when it generates lift power, a pair of vortices in opposite directions will be derived behind it. The relevant variables of the abovementioned reverse vortex pair can be calculated with the relevant performance data of the aircraft (maximum take-off weight, flight speed, wingspan, etc.). As a result, the calculation of wake development and evolution has been simplified. And this theory and hypothesis have been widely used in the study of wake analysis.

$$
\begin{gathered}
b_{0}=\frac{\pi}{4} b, \\
r_{c}=005 b_{0}, \\
\Gamma_{\infty}=\frac{W_{T_{0}} g}{\rho \cup b_{0}},
\end{gathered}
$$

where $b_{0}$ is the distance between the left and right vortices; $b$ is the wingspan length; $r_{c}$ is the vortex core radius; $\Gamma_{\infty}$ is the vortex ring volume; $W_{T_{0}}$ is the maximum take-off weight; $\rho$ is the air density; $U$ is the acceleration of gravity; $U$ is the velocity of the incoming vortex.

2.2.2. Convection-Diffusion Equation. The convection-diffusion equation is a nonlinear equation used to characterize convection and diffusion. And it is also a kinematic 
equation, which is generally used in areas where the wake velocity is relatively stable, such as near and middle wakes. The mathematical expression when using the convectiondiffusion equation for time-domain simulation is as follows [35]:

$$
\left\{\begin{array}{l}
\frac{\partial s}{\partial t}+u_{i} \frac{\partial s}{\partial x_{i}}-D \frac{\partial^{2} S}{\partial x_{2}^{2}}=0 \\
S[y \widehat{y}, 0]=y_{0}-y
\end{array}\right.
$$

where $u_{i}$ is the rotational velocity field in the wake; $D$ is the diffusion coefficient of air; $S$ is the conservative passive quantity of the solution of the equation; $[y \widehat{y}, 0]=y_{0}-y$ is the initial condition.

\subsection{Numerical Simulation of Aircraft Wake Based on CFD.} In order to make up for the difficulties such as high cost, long research time, and low accuracy of the test methods, the CFD method began to be applied to the analysis and prediction of the wake vortex field. In the 1960s, Takami used the vortex plate method for the numerical simulation of the wake, which can obtain the induced velocities at various locations in the vortex field [36]. Scholars visualized the time-based simulation and formed a wake model that can vary with a scale, which officially started the real three-dimensional wake simulation [37]. When the Reynolds average N-S equation appeared and began to be applied, CFD numerical methods began to be used to simulate the three-dimensional vortex field from the generation, development, and dissipation of wake [38]. Recent studies have shown a variety of numerical simulation methods, including large eddy simulation, which show good vortex characteristics for wake simulations, that is in good compliance with some phenomena observed in experiments [39].

In addition, the CFD numerical simulation method can also achieve faster simulation by combining classic wake models without the excessive pursuit of calculation accuracy. The initial wake vortex field distribution data obtained by the wake model can further develop the numerical evolution, which can predict the safety zone of the wake [39]. On the basis of retaining a certain calculation accuracy, the calculation efficiency is greatly improved, which is of great significance to the calculation and pre diction of wake interval. The CFD method has unique advantages such as short calculation time, low cost, easy data extraction, and the continuous optimization and improvement of CFD technology, making it the main direction of fluid mechanics research.

\section{CFD Modeling of Aircraft Wake Vortex Field}

In order to analyze the motion characteristics of the wake vortex field of the paired aircrafts, the CFD method is used to construct the wake vortex field model of the paired aircrafts. The numerical simulation process of the wake vortex field is completed through preprocessing of the model calculation and setting of boundary conditions. Finally, by postprocessing the numerical calculation results, the wake vortex field of the paired aircrafts can be quantitatively analyzed.

\subsection{Model Construction of Aircraft Wake Vortex Field.} The construction of the aircraft wake vortex field model is an important part of the numerical simulation process. The density, quantity, and quality of the grid cells in the built model will directly affect the accuracy of the numerical simulation. The construction of the aircraft wake vortex field model mainly includes the following steps: establishment of the aircraft wing geometric model, construction and design of the aircraft wake vortex field calculation domain, and model calculation preprocessing (mesh division, dense calculation domain, setting boundary conditions).

3.1.1. Geometric Modeling of the Lead Aircraft Wing. In view of the extremely complex configuration of the aircraft wing, the entire wing is composed of several parts connected together. In addition to the main wing, there are multiple structures such as flaps, ailerons, spoilers, winglets, and engine pods. If the real wing structure is to be simulated numerically, it is not only difficult to model but also difficult to realize numerical simulation with the existing computer technology and CFD technology. Therefore, this paper simplifies the actual physical configuration of the wing. Based on the standard model of Boeing's transport airliner, the modeling tool SolidWorks is used to intercept and construct the wing geometric model. According to the actual geometric size of the paired aircrafts, the model is scaled down to obtain a suitable geometrical model of the wing. Taking the B747-400 wing as an example, the wingspan of the B747-400 is $211 \mathrm{ft}$, and the reduced geometric model of the B747-400 wing is shown in Figure 3. During an aircraft approach, it usually flies at a certain angle of attack, and a certain angle of attack can be set when the model is built.

3.1.2. Calculation Domain Setting of Aircraft Wake Vortex Field. The computational domain of the vortex field refers to the mathematical operation (usually integral operation) during the numerical simulation calculation process. There are two ways to generate the computational domain-direct modeling and geometric extraction. In this paper, we need to study the wake vortex field of aircraft. The scale of the wake vortex is relatively large, so it is obviously impossible to generate the computational domain through geometric extraction. It is more appropriate to adopt direct modeling. In the actual calculation, in order to reduce the calculation load and the calculation pressure, the geometry of the calculation domain is usually simplified. Using the symmetry of the geometric model and the periodicity of fluid flow are common geometric simplification methods. For the numerical simulation of the external vortex field of the aircraft, the experimenter usually constructs the computational domain as a cylinder. In order to reduce the amount of calculation and take into account the motion characteristics of the wake, this paper simplified the calculation domain of 


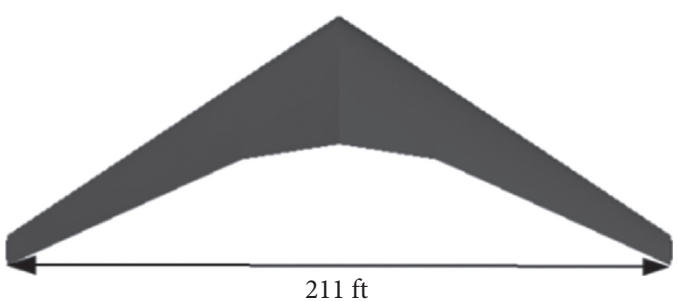

FIgURE 3: Geometric model of B747-400 wing.

the outer vortex field of the aircraft to the truncated cone shown in Figure 4. The calculation domain of the outer vortex field is divided into INLET, OUTLET, and WALL.

3.1.3. Calculation Model Preprocessing. The preprocessing process of the model generally includes meshing of the vortex field model, compacting the computational domain, and setting the boundary conditions. Compared to the construction and design of the computational domain, meshing is more important. The proper processing of the computational grid and the quality of grid generation are the primary conditions for numerical simulation calculations. The selection of grid quantity is generally based on empirical value or reference to the recommendations of relevant literature. In this paper, we use ANSYS ICEM CFD for preprocessing. It can provide advanced grid generation, geometry acquisition, and grid cell optimization functions for the analysis of complex models. It can also output grids for fluid mechanics solvers. The modeling tool SolidWorks will be used to intercept and build the wing geometric model. Then, it is imported into ANSYS ICEM CFD, the geometric model is checked, geometric repair is performed, and the above-mentioned calculation domain is generated. Then, the appropriate grid algorithm is selected to generate the computational grid of the geometric wing model and the computational domain, that is, the computational grid of the wake vortex field model. There are three grid algorithms provided by ANSYS ICEM CFD such as Octree algorithm, Delaunay algorithm, and Advance Front algorithm. The octree algorithm is commonly used for the formation of unstructured grid tetrahedron. This algorithm is more intelligent and automatic, so it was chosen for grid generation in this paper. In order to save computing resources and time, an unstructured grid is selected in this paper. The process of using ANSYS ICEM CFD for meshing is shown in Figure 5 in detail. First, whole grid cells are set, including grid parameters, plane grid, volume grid, prism layer, and period. Then, grid encryption is carried out for the computing domain by setting partial grid, plane grid, and line grid, and creating grid density region. Finally, meshing these grids, including three parts: meshing plane grid, meshing volume grid, and meshing prism layer grid. After the above steps, the wake vortex field of the paired aircrafts was meshed. The mesh model of the wing geometry model and the computational domain mesh model after the division are shown in Figure 6 and Figure 7, respectively. The $X$-axis indicates the direction of the incoming vortex, the $Y$-axis indicates the span direction, and the $Z$-axis indicates the height. The total

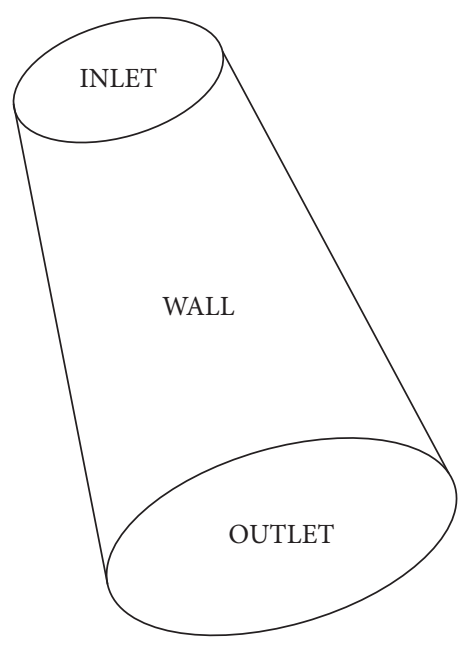

Figure 4: Computational domain of aircraft wake vortex field.

grid quantity of the wake vortex field is about $14,000,000$. Taking into account the principle of the wake generation during aircraft flight, the leading edge of the wing needs to be arranged with a dense grid so as not to affect the calculation accuracy. In order to capture the wingtip vortices, the computational domain grid of the area where the wake is formed downstream of the aircraft should also be for refinement processing. The grid division details of the leading edge of the wing are shown in Figure 8, and the details of computational domain grid refinement are shown in Figure 9.

For the finished grid, it cannot be directly used for calculation but must go through grid output setting and grid quality check. The grid quality is related to the calculation precision and also determines whether the calculation can converge. Check and Report Quality functions in ANSYS ICEM CFD can be used to check the grid. It mainly detects the geometric size, volume, and grid proportion of the mesh model, among which the minimum volume parameter needs to be paid more attention, and its value must be positive. When the grid model has a negative volume, it cannot be used for numerical simulation calculations. It is generally believed that the grid slope is better than 0.3. the Check and Report Quality functions are used in ANSYS ICEM CFD software to conduct a quality check on the grid model. The quality check result of the grid is shown in Figure 10. A small part of the grid has a slope of less than 0.3 , and there is no negative volume grid. It is considered to meet the requirements of numerical simulation calculation.

\section{Calculation of Wake Separation Based on CFD}

There are two safe areas in the paired approach mode. They are the first safe area (the safety zone mentioned in Section 2) and the second safe area. This article focuses on the first safe area in the paired approach mode. The first safe area is the maneuvering flight area that is between the CSL and WSL of the paired aircrafts. The second safe area is an area where the wake of the lead aircraft has dissipated. Due to the 


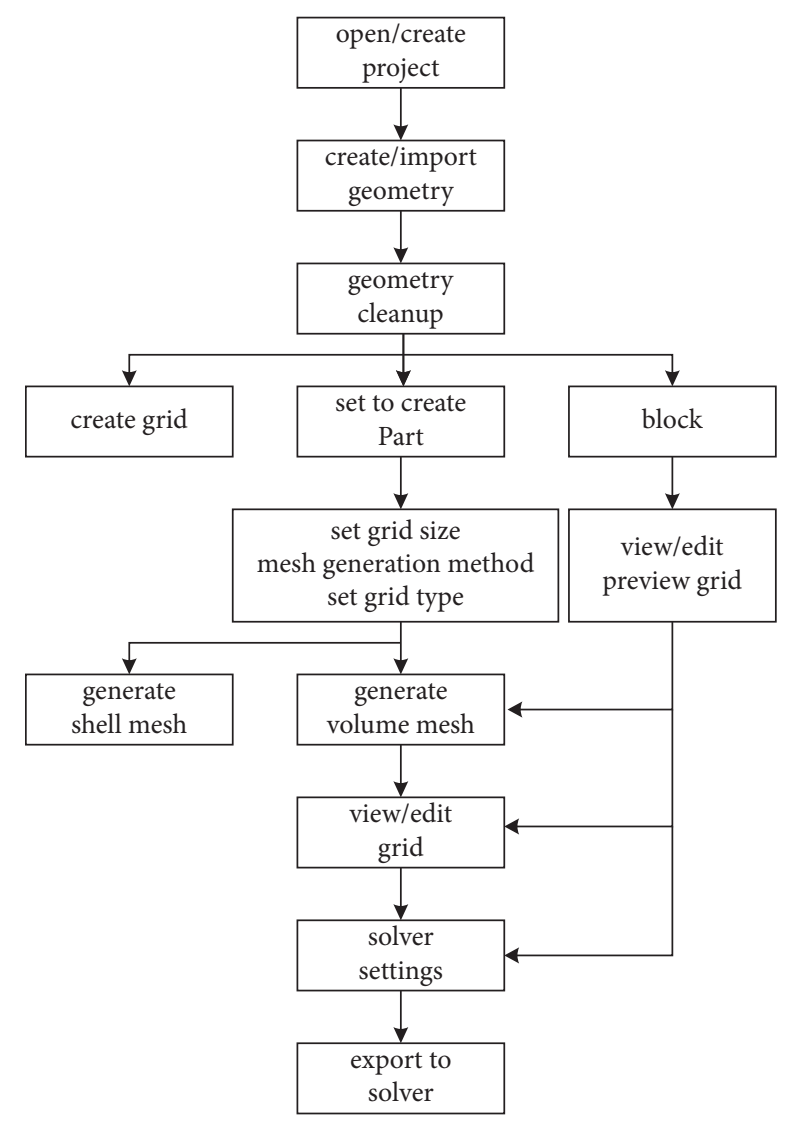

FIGURE 5: ANSYS ICEM CFD meshing process.

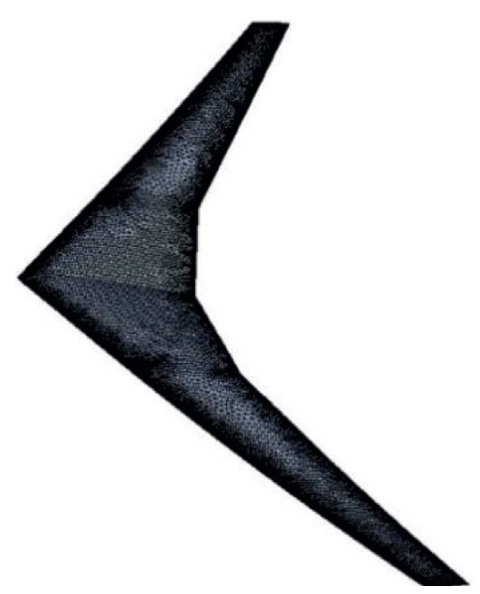

FIGURE 6: Mesh model of the wing geometry model.

development of communication, navigation, and surveillance equipment, the CSL caused by the wrong approach of the paired lead aircraft can basically be ignored. Therefore, the research on the first safe area (safety zone) of the paired approach mode can focus on the WSL which needs to calculate the maximum wake safety interval.

The CFD-based wake safety interval calculation method combines knowledge and technology in the fields of fluid mechanics, mathematics, and computer science. The purpose of this method is to make up for the difficulty of the test
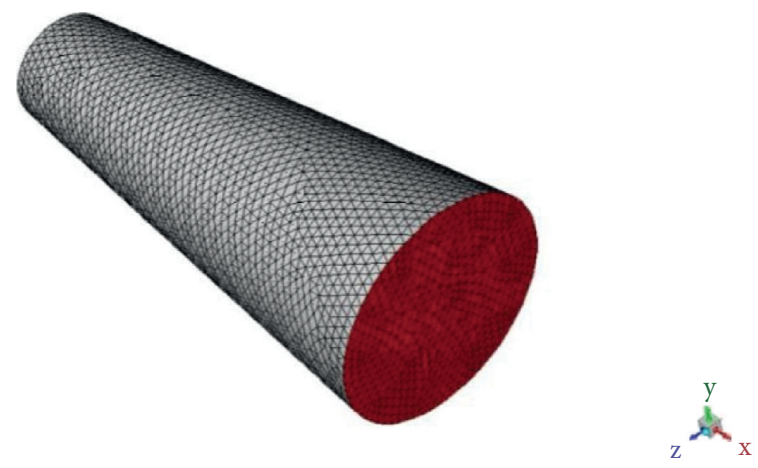

Figure 7: Computational domain grid model.

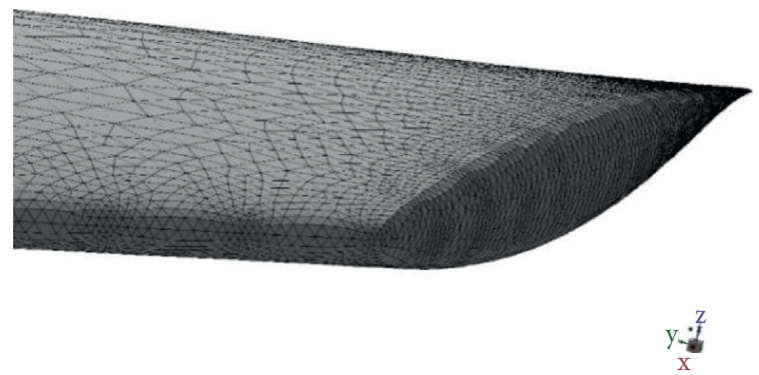

Figure 8: Details of the meshing lead edge of the wing.

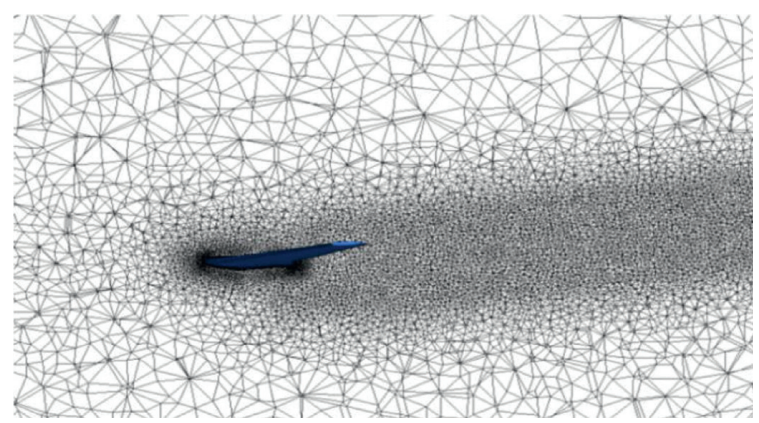

FIgURE 9: Detailed diagram of computational domain grid encryption.

method, the high cost, and the long research period. It can also make up for the shortcomings of using the wake model method to simulate complex wake vortex fields with insufficient accuracy. The calculation of the wake safety separation tends to be simplified under the premise of ensuring a certain calculation accuracy.

4.1. Determining the Initial Lateral Interval of the Paired Aircraft. For the $3^{\circ}$ offset paired approach mode, before the trailing aircraft reaches the wake protection point, there is always a vertical safety gap of $1,000 \mathrm{ft}$ between the two aircrafts, so the trailing aircraft does not need to consider the impact of the wake of the lead aircraft. When the trailing aircraft reaches the wake protection point, since the paired aircrafts do not need to maintain a vertical separation of 


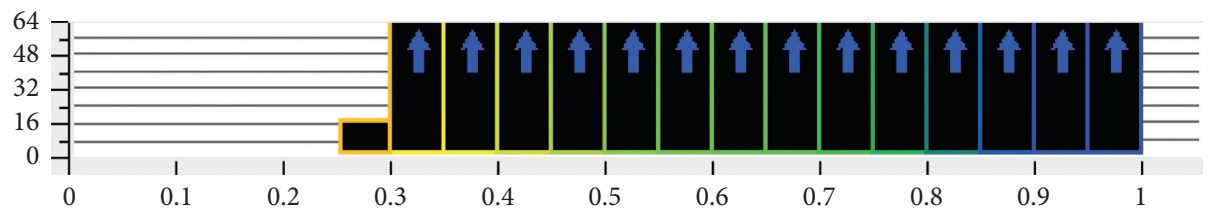

FIgURE 10: Grid quality inspection results.

$1,000 \mathrm{ft}$ at this time, the trailing aircraft must consider avoiding the influence of the wake of the lead aircraft. For the straight-in paired approach mode, since the distance between the runway centerlines of CSPRs is less than $2500 \mathrm{ft}$, a safe wake distance between the two aircraft should be determined at the beginning of the pairing to ensure flight safety. At this time, the initial lateral separation between the two aircraft is the distance between the centerline of the runway $(C)$. Since the wake of the lead aircraft is generated near the wingtip, in order to make the calculation result more conservative, one half of the wingspan of the rear aircraft $\left(B_{2}\right)$ should be deducted from the runway centerline spacing $\left(D_{0}\right)$ as the starting side of the paired aircraft which is shown in Figure 11.

4.2. Numerical Simulation of the Lead Aircraft's Wake Vortex Field. According to the methods and steps described above, the wake vortex field of the lead aircraft is numerically simulated. The result file is obtained after the numerical simulation calculation converges. The result file of the numerical simulation of the lead aircraft wake vortex field is postprocessed to obtain a visual lead aircraft wake vortex field (vorticity cloud map, vorticity isosurface map, etc.), as shown in Figure 12. Combining the basic process of the paired approach mode, the CFD-based paired approach wake safety interval calculation model is constructed.

Wing-tip vorticity, which is the wake of aircraft during flight, can be identified by vorticity. In TecPlot and CFDPost, the variable vorticity needs to be customized. For example, in CFD-Post, new expressions and new variables can be created, and the two corresponding velocity differential variables can be loaded successively. The variable vorticity can be obtained by subtraction operation, and then the vorticity cloud map can be obtained. In TecPlot, the variable vorticity can also be obtained by creating a vorticity calculation formula in a similar way. The specific formula is as follows [24]:

$$
\begin{aligned}
& \{w x\}=d \mathrm{~d} y(\{w\})-d \mathrm{~d} z(\{v\}), \\
& \{w y\}=d \mathrm{~d} z(\{u\})-d \mathrm{~d} z(\{v\}), \\
& \{w z\}=d \mathrm{dx}(\{v\})-d \mathrm{~d} z(\{v\}) .
\end{aligned}
$$

4.3. Determining the Wake Safety Interval. As mentioned in the previous section, the strength and direction of the vortex can be described by the vorticity, so this article uses the vorticity as the physical quantity to identify the range of the wake vortex field. The paired approach mode requires the trailing aircraft to avoid the wake of the lead aircraft before

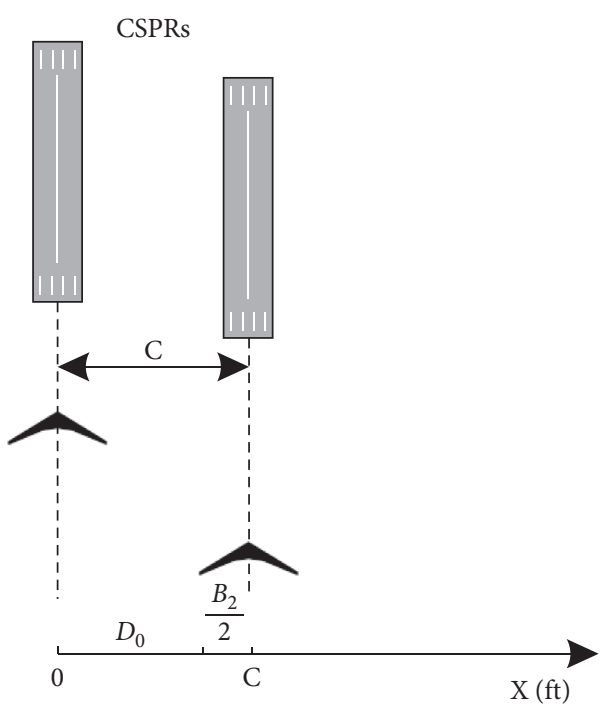

Figure 11: The initial lateral interval of the paired aircrafts.

the wake of the lead aircraft arrives so as to keep the trailing aircraft always maneuvering in the first safety zone. Therefore, according to the CFD-based paired approach wake safety interval calculation model, the maximum wake safety interval between the paired aircrafts can be obtained. The wake safety interval $(D)$ at this time is obtained from the vorticity distribution on the XY section $\left(Y=D_{0}\right)$ of the lead aircraft in the downwind direction. Assuming that the vorticity is on the XY section of the downwind direction of the paired aircraft, at the time $Y=D_{0}$ and point $X=D_{1}$, the absolute value is greater than 0 , and the wake safety interval (D) of the paired aircraft can be obtained as follows:

$$
D=D_{1} \text {. }
$$

4.4. The Limitation of Present Work. This model can provide some theoretical reference for the operation of paired approach mode. However, the following aspects need further discussion and research: the wing model of the paired lead aircraft established in this paper is the standard wing model of transport aircraft. In future research, a specific model can be developed for each type of aircraft, to further reduce the error between numerical simulation test and real value. When constructing the calculation model of safe wake interval based on CFD, it is assumed that the distance between two aircrafts is the maximum safe wake interval when the absolute value of vorticity at the wing of the trailing aircraft is not zero, which is just to simplify the 


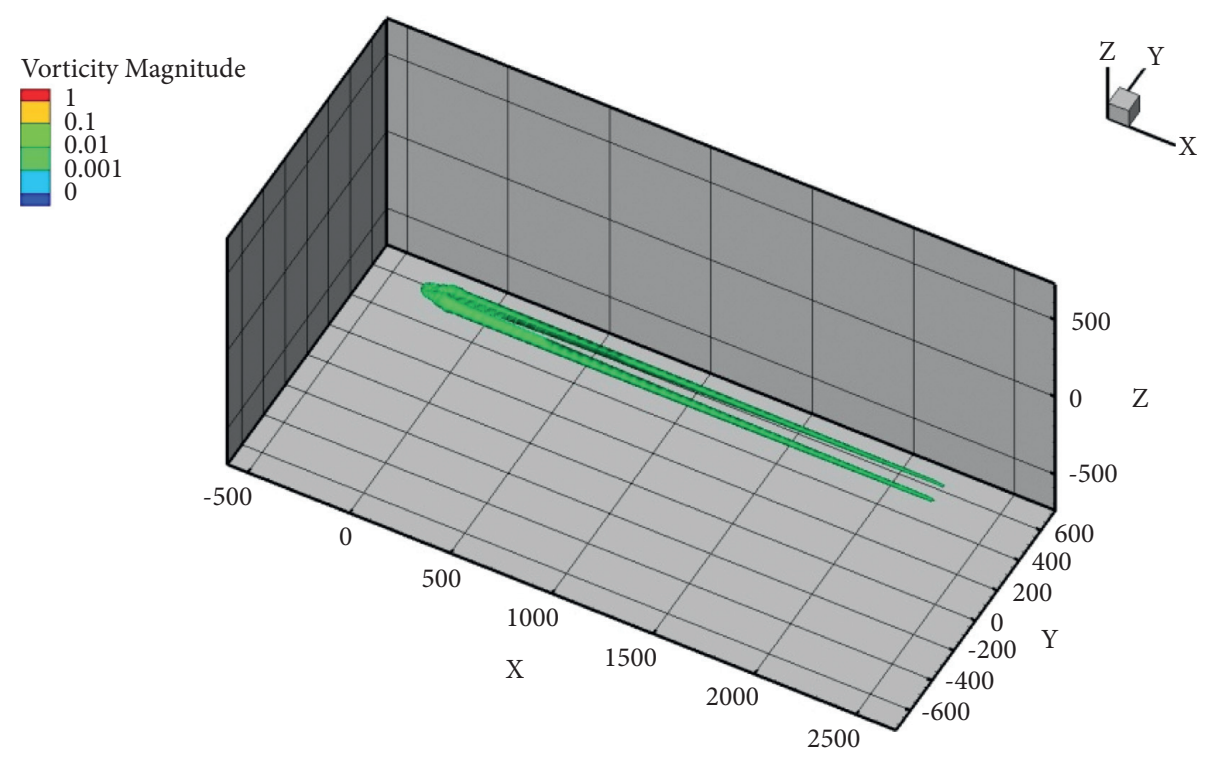

FIGURE 12: Isosurface map of the tail vortex of the paired aircraft with a vortex of 0.01 under static wind conditions.

calculation. In the future, the trailing aircraft can be assumed as a cuboid or ellipsoid to make it a more reasonable model.

\section{Conclusion}

In this paper, the computational fluid dynamics method is used to model the wake separation optimization when two aircrafts are operating in the paired approach mode. Through the theory introduction, modeling introduction in detail, and numerical simulation and analysis concluded that using the CFD method to study the safety zone of the paired approach wake is feasible. Comparing with experimental methods-wind tunnel test and water tunnel test, the CFD numerical simulation method shows great superiority. The research results of this paper can provide a certain theoretical reference for the study of the wake safe area of the paired approach mode and provide certain theoretical support for the specific implementation of the paired approach mode in domestic air transportation.

This method also has some shortcomings that need to be improved. This method only considered the first safe area, ignoring the collision risk caused by the lead aircraft when it makes a wrong approach and intrudes in the trailing aircraft's course. This would be the future research direction of the study. Also, a comparative study between experimental methods and CFD would be considered in the follow-up study.

\section{Data Availability}

The data used to support the findings of this study are available from the corresponding author upon request.

\section{Conflicts of Interest}

The authors declare that they have no conflicts of interest.

\section{Acknowledgments}

This research was supported by the Key Laboratory of Civil Aviation Flight Technology and Safety, CAAC Aviation Safety Capacity Building Fund Supported Project and CAAC Air Traffic Management Bureau Project.

\section{References}

[1] F. Holzäpfel, T. Gerz, F. Köpp et al., "Strategies for circulation evaluation of aircraft wake vortices measured by lidar," Journal of Atmospheric and Oceanic Technology, vol. 20, no. 8, pp. 1183-1195, 2003.

[2] M. L. Williams, L. C. Wood, and B. J. Nelson, "Safety study of closely spaced parallel operations utilizing paired approach," in Proceedings of the2019 IEEE/AIAA 38th Digital Avionics Systems Conference (DASC), pp. 1-10, IEEE, San Diego, CA, USA, September 2019.

[3] M. C. Waller and C. H. Scanlon, "Flight deck centered parallel runway approaches in instrument meteorological conditions," in Proceedings of the NASA workshop on flight deck centered parallel runway approaches in instrument meteorological conditions, Washington, DC, USA, October 1996.

[4] S. J. Landry and A. R. Pritchett, "Incorporating procedural information in a paired approach task," Proceedings of the Human Factors and Ergonomics Society - Annual Meeting, SAGE Publications Sage CA, vol. 46, no. 1, pp. 106-110, 2002.

[5] R. R. Eftekari, J. B. Hammer, D. A. Havens, and A. D. Mundra, "Feasibility analyses for paired approach procedures for closely spaced parallel runways,"in Proceedings of the IntegratedCommunications, Navigation, and Surveillance Conference Proceedings, IEEE, Herndon, VA, USA, May 2011.

[6] J. Hammer, "Case study of paired approach procedure to closely spaced parallel runways," Air Traffic Control Quarterly, vol. 8, no. 3, pp. 223-252, 2000.

[7] R. Bone, O. Olmos, A. Mundra, and R. Jensen, Paired Approach: A Closely Spaced Parallel Runway Approach Concept, Federal Aviation Administration, Washington, DC, USA, 2000. 
[8] R. Teo, J. S. Jang, and C. Tomlin, "Flight demonstration of provably safe closely spaced parallel approaches," in Proceedings of the AIAA Guidance, Navigation and Control Conference and Exhibit, p. 6197, San Francisco, CA, USA, August 2005.

[9] R. Bone, A. Mundra, and B. O. Olmos, "Paired approach operational concept," in Proceedings of the 20th DASC. 20th Digital Avionics Systems Conference(Cat. No. 01CH37219), vol. 1, pp. 5B3/1-5B3/14, IEEE, Daytona Beach, FL, USA, October 2001.

[10] A. Pritchett, S. Landry, and A. Pritchett, "Two studies of paired approaches," in Proceedings of the Air Traffic Management Research and Design Workshop, Sante Fe, NM, USA, November 2001.

[11] B. McKissick, "Wake encounter analysis for a closely spaced parallel runway paired approach simulation," in Proceedings of the 9th AIAA Aviation Technology, Integration, and Operations Conference (ATIO) and Aircraft Noise and Emissions Reduction Symposium, p. 6943, Hilton Head, SC, USA, October 2009.

[12] N. Guerreiro, K. Neitzke, S. Johnson, H. Stough, B. McKissick, and H. Syed, "Characterizing a wake-free safe zone for the simplified aircraft-based paired approach concept," in Proceedings of the AIAA Atmospheric and Space Environments Conference, p. 7681, Toronto, Canada, August 2010.

[13] M. M. Madden, "Kinematic modeling of separation compression for paired approaches to closely-spaced parallel runways," in Proceedings of the 14th AIAA Aviation Technology, Integration, and Operations Conference, p. 3150, Atlanta, GA, USA, June 2014.

[14] S. C. Johnson, G. W. Lohr, B. T. McKissick, N. M. Guerreiro, and P. Volk, "Simplified aircraft-based paired approach: concept definition and initial analysis," National Aeronautics and Space Administration, Langley Research Center, Hampton, VA, USA, 2013.

[15] R. B. Perry, M. M. Madden, W. Torres-Pomales, and R. W. Butler, The Simplified Aircraft-Based Paired Approach with the ALAS Alerting Algorithm, Citeseer, 2013.

[16] S. Priess, "Analysis of an ADS-B in method for calculating the interval management paired approach collision safety limit," in Proceedings of the 2017IEEE/AIAA 36th Digital Avionics Systems Conference (DASC), pp. 1-8, IEEE, November 2017.

[17] N. A. Durkins, Aircraft Type Designators, Department of Transportation Air Traffic Organization Policy, FAA. JO 7360.1F, U.S, 2021.

[18] K. Leiden, S. Priess, P. Harrison, R. Stone, P. Strande, and M. Palmer, "Paired approach flight demonstration: planning and development activities," in Proceedings of the 2018 Integrated Communications, Navigation, Surveillance Conference (ICNS), pp. 3G4-1-3G4-12, IEEE, Herndon, VA, USA, April 2018.

[19] H. Dai, D. I. Dongning, H. Qiao, and J. Cui, "Research progress and application prospects of aircraft wake detection technology," Science and Technology Review, vol. 31, no. 31, pp. 66-69, 2013.

[20] A. C. De Bruin, G. Hegen, P. B. Rohne, and P. R. Spalart, "Flow field survey in trailing vortex system behind a civil aircraft model at high lift," in Proceedings of the Characterization \& Modification of Wakes from Lifting Vehicles in Fluids, AGARD FDP Symposium, pp. 25-1-25-12, Trondheim, Norway, May 1996.

[21] A. C. De Bruin and R. Oerlemans, Flow Field Measurement Data Presentation and Analysis System for NLR Rake with 5-hole Probes, NLR TR 96540 L, 1996.
[22] H. Vollmers, "Detection of vortices and quantitative evaluation of their main parameters from experimental velocity data," Measurement Science and Technology, vol. 12, no. 8, pp. 1199-1207, 2001.

[23] D. L. Ciffone, "Vortex interactions in multiple vortex wakes behind aircraft," Journal of Aircraft, vol. 14, no. 5, pp. 440-446, 1977.

[24] K. Huenecke, "Wake vortex investigations of transport aircraft," in Proceedings of the 13th Applied Aerodynamics Conference, p. 1773, San Diego, CA, USA, June 1995.

[25] K. Huenecke, "Structure of a transport aircraft-type near field wake," in Proceedings of the Characterization \& Modification of Wakes from Lifting Vehicles in Fluids, pp. 5-1-5-9, AGARD FDP Symposium, Trondheim, Norway, April 1996.

[26] K. Huenecke, "Vortex wakes from large aircraft-A challenge for industrial research," in Proceedings of the Fluids 2000 Conference and Exhibit, p. 2216, Denver, CO, USA, June 2000.

[27] K. Hünecke, "Wake vortex control-a challenge for large transport aircraft," Air \& Space Europe, vol. 3, no. 3-4, pp. 209-213, 2001.

[28] K. Huenecke, "The characterisation of transport aircraft vortex wakes," in Proceedings of the 19th AIAA Applied Aerodynamics Conference, p. 2427, A, June 2001.

[29] L. Jacquin, D. Fabre, P. Geffroy, and E. Coustols, The properties of a transport aircraft wake in the extended near field-An experimental study, 39th Aerospace Sciences Meeting and Exhibit, Reno, NV, 2001.

[30] C. Breitsamter, "Nachlaufwirbelsysteme großer transportflugzeuge-experimentelle charakterisierung und beeinflussung (wake-vortex systems of large transport aircraft-experimental characterization and manipulation)," Inaugural Thesis, Technische Universität München, München, Germany, 2007.

[31] V. J. Rossow and L. S. A. Branch, "Measurements in vortex wakes shed by conventional and modified subsonic aircraft," in Proceedings of the Characterization \& Modification of Wakes from Lifting Vehicles in Fluids, pp. 26-1-26-9, AGARD FDP Symposium, Trondheim, Norway, November 1996.

[32] B. R. Cobleigh and J. Del Frate, Water Tunnel Flow Visualization Study of a 4.4\% Scale X-31 Forebody, Citeseer, 1994.

[33] S. E. Sanders, O. R. Willis, N. H. Nahler, and E. Wrede, "Absolute fluorescence and absorption measurements over a dynamic range of 106with cavity-enhanced laser-induced fluorescence," The Journal of Chemical Physics, vol. 149, no. 1, Article ID 014201, 2018.

[34] J. Kang, Y. R. Wang, R. H. Li, and Y. Q. Chen, "Surface elemental microanalysis with submicron lateral resolution by the laser-ablation laser-induced fluorescence technique," Optics Express, vol. 26, no. 11, Article ID 14689, 2018.

[35] T. J. Myers, Determination of Bragg Scatter in an AircraftGenerated Wake Vortex System for Radar Detection, Virginia Polytechnic Institute and State University, Blacksburg, Virginia, 1997.

[36] H. Takami, A Numerical experiment with Discrete Vortex Approximation with Reference to the Rolling up of a Vortex Sheet, Stanford Univ. Report Sudaer, Stanford, CA, US, 1964.

[37] D. C. Lewellen and W. S. Lewellen, "The effects of aircraft wake dynamics on contrail development," Journal of the Atmospheric Sciences, vol. 58, no. 4, pp. 390-406, 2001.

[38] E. Coustols, L. Jacquin, and G. Schrauf, "Status of wake vortex alleviation in the frame work of European collaboration: 
validation attempts using tests and CFD results," in Proceedings of the European Conference on Computational Fluid Dynamics, ECCOMAS CFD, Netherlands, January 2006.

[39] L. Mengda, C. Guixiang, Z. Zhaoshun, X. Chunxiao, and H. Weixi, "Large eddy simulation on the evolution and the fast-time prediction of aircraft wake vortices," Journal of mechanics, vol. 49, no. 6, pp. 1185-1200, 2017. 